\title{
How to optimize culture media osmolality during Assisted Reproductive Technologies treatments
}

\author{
Renata de Lima Bossi ${ }^{1}$, Brenda Campos Villa Pinto ${ }^{1}$, Marcos Aurelio Coelho Sampaio ${ }^{1}$, Selmo Geber ${ }^{1}$ \\ ${ }^{1}$ Centro de Medicina Reprodutiva - ORIGEN, Belo Horizonte, MG, Brazil
}

The results were presented at the $76^{\text {th }}$ ASRM annual meeting

\begin{abstract}
Objective: The objective of our study was to compare the osmolality in sequential and single step culture media, used for in vitro human embryo culture, covered with mineral oil and paraffin, in dry and humid incubators.

Methods: We performed a prospective observational study. A total of 120 Petri dishes, with 960 droplets of culture media, were evaluated. Each dish was prepared with 4 droplets of single step medium and sequential medium. Sixty dishes were covered with mineral oil and 60 with paraffin oil. Half were incubated in a dry incubator and half in a humid. Osmolality was measured on days 1 , $3,5,7$. ANOVA test was performed for statistical analysis.

Results: Osmolality results for single step and sequential medium, that were covered with both mineral and paraffin oil and placed in the dry incubator, significantly increased throughout the study time (D7>D5>D3). In the humid incubator, the results were similar for all periods. Osmolality was significantly lower in humid incubator, in all periods, when droplets were covered with both oils. When both culture media were placed in the humid incubator, no variation was detected, using both oils. However, when single step medium was placed in the dry incubator, covered with mineral oil, we observed a higher osmolality than the covered with paraffin oil.

Conclusions: TWe can conclude that humid incubator is better for maintaining osmolality and paraffin oil protect single step media from evaporation in dry incubator.
\end{abstract}

Keywords: osmolality, culture medium, incubator, oil, human assisted reproduction

\section{INTRODUCTION}

Improvements in embryo culture protocols have allowed significant increase in ART treatment outcomes. Several types of culture media, oils, disposable materials and incubation systems have been exhaustively tested in the last 40 years (Swain, 2019). Some essential variables to consider within the culture system that include $\mathrm{pH}$, temperature, gas concentration, osmolality and air quality, must be controlled to improve embryo development (Eaton et al., 2012; Swain et al., 2012; Heitmann et al., 2015; Swain, 2019).

Osmolality is a measure of solute particles dissolved in a solution calculated by an osmometer and is a well-known cell stressor that can impact embryo development. Human embryo development occurs in culture media under an osmolality range of 255-295 mOsm/kg (Erstad, 2003; Richards et al., 2010). Some variables such as preparation time, droplet size, temperature and air flow can impact evaporation during culture dish preparation, that can change culture media osmolality (Swain et al., 2012).

Culture media used for in vitro human embryo development is composed by aminoacids, salts, proteins, ions, water and a source of energy (pyruvate and glucose). Several different commercial culture media are available for ART treatments that can be for sequential or single step media (Bolton et al., 2014; Morbeck et al., 2014). Sequential media are based on two different formulations: one for the cleavage stage period (day1-day3), and the other for blastocyst stage (day 4-day6) (Gardner \& Lane, 1997). Single step media is formulated to allow embryo development from day 1 to day 6 (Biggers \& Racowsky, 2002).

Also, different types of oil can be used to cover the culture media droplets and minimize evaporation and $\mathrm{pH}$ fluctuations. Regardless the type of oil used, evaporation will occur. Therefore, the type of oil used, directly affects the speed of evaporation and heavy oils seems to provide less evaporation compared to a lighter oil (Kovačič, 2012; Swain, 2018). Paraffin and mineral oil are known to have different chemical properties, as mineral oil is lighter than paraffin (Otsuki et al., 2007). As mineral oil contains more unsaturated bonds than paraffin oil, it is more sensitive to photooxidation and peroxidation that is more detrimental to fertilization and embryo development (Elder et al., 2015). Some studies showed that use of paraffin oil results in less evaporation and higher incidence of good quality embryos (Sifer et al., 2009; Yumoto et al., 2018).

Additionally, the type of incubator, i.e., humid or dry, may also affect embryo development as it can interfere with contamination and evaporation rate of the culture media (Mori et al., 2010; Swain, 2014). Moreover, incubator oxygen level is crucial for better results in IVF cycles (Catt \& Henman, 2000) as low oxygen concentration may enhance blastocyst development (Gardner \& Lane, 1997; Bontekoe et al., 2012; Kovačič, 2012). Incubators with 5\% of oxygen resulted in better results should be of reducing oxidation levels and maintaining the integrity of the amino acids in culture media (Biggers et al., 2004; Tarahomi et al., 2019).

Therefore, the aim of our study was to compare the impact of the type of culture media, type of oil used to cover the culture media and the type of incubator on the osmolality, during the embryo culture period.

\section{MATERIALS AND METHODS}

We performed a prospective observational study between March and November 2019 to evaluate the osmolality of two different types of culture media covered by two different types of oil, placed in two types of incubator. As we did not use biological material an institutional review board approval was not necessary.

A total of $425 \mu \mathrm{l}$ droplets of single step medium (CSCM-C, Irvine Scientific, USA) and $425 \mu$ droplets of sequential medium (Sydney IVF Cleavage Medium, Cook, USA) were placed in $12035 \times 10 \mathrm{~mm}$ Petri dishes (Falcon, USA), both media in same dish. From those, 60 were covered by $3 \mathrm{~mL}$ of paraffin oil (Ovoil, Vitrolife, Sweden) 
and 60 by $3 \mathrm{~mL}$ of mineral oil (Light Mineral Oil, Irvine Scientific, USA). For each subgroup, 30 were placed in humid water jacket (Forma 4130, Thermo Scientific) and 30 in dry bench top (G185, K-Systems), both Tri-gas with $5 \% \mathrm{O}_{2}, 9.0 \% \mathrm{CO}_{2}, 86 \% \mathrm{~N}_{2}$, at the same temperature. All preparations were performed by the same person, using the same material, equipment, calibration, at the same temperature $\left(23^{\circ} \mathrm{C}\right)$ and time of the day.

Weekly 20 Petri dishes were prepared, one by one, under a laminar flow hood at room temperature, using 25 microliter droplets of both culture media and covered with $3 \mathrm{~mL}$ of oil, mineral or paraffin. Osmolality $(\mathrm{mOsm} / \mathrm{kg})$ was measured on days $1,3,5$ and 7 after preparation (one droplet per day) of each medium, covered with paraffin or mineral oil, placed in dry or humid incubator, using the same osmometer (Advanced Instruments 3320, USA), at the same time of the day and by the same embryologist. After collect the droplet for osmolality analysis dishes were replaced in same incubator until next measurement. Briefly, weekly we placed in dry incubator 5 dishes with $425 \mu$ l droplets of CSCM-C $+425 \mu$ d droplets of Cleavage Medium covered with mineral oil and 5 dishes with $425 \mu$ droplets of CSCM-C $+425 \mu$ l droplets of Cleavage Medium covered with paraffin. In humid incubator we placed 5 dishes with $425 \mu$ l droplets of CSCM-C $+425 \mu$ l droplets of Cleavage Medium covered with mineral oil and 5 dishes with $425 \mu \mathrm{l}$ droplets of CSCM-C $+425 \mu$ ldroplets of Cleavage Medium covered with paraffin.

Therefore, in the end, we had eight groups: Group 1 (CSCM-C with mineral oil in dry incubator), Group 2 (CSCM-C with mineral oil in humid incubator), Group 3 (CSCM-C with paraffin oil in dry incubator), Group 4 (CSCM-C with paraffin oil in humid incubator), Group 5 (Cleavage with mineral oil in dry incubator), Group 6 (Cleavage with mineral oil in humid incubator), Group 7 (Cleavage with paraffin oil in dry incubator), Group 8 (Cleavage with paraffin oil in humid incubator) (Figure 1).

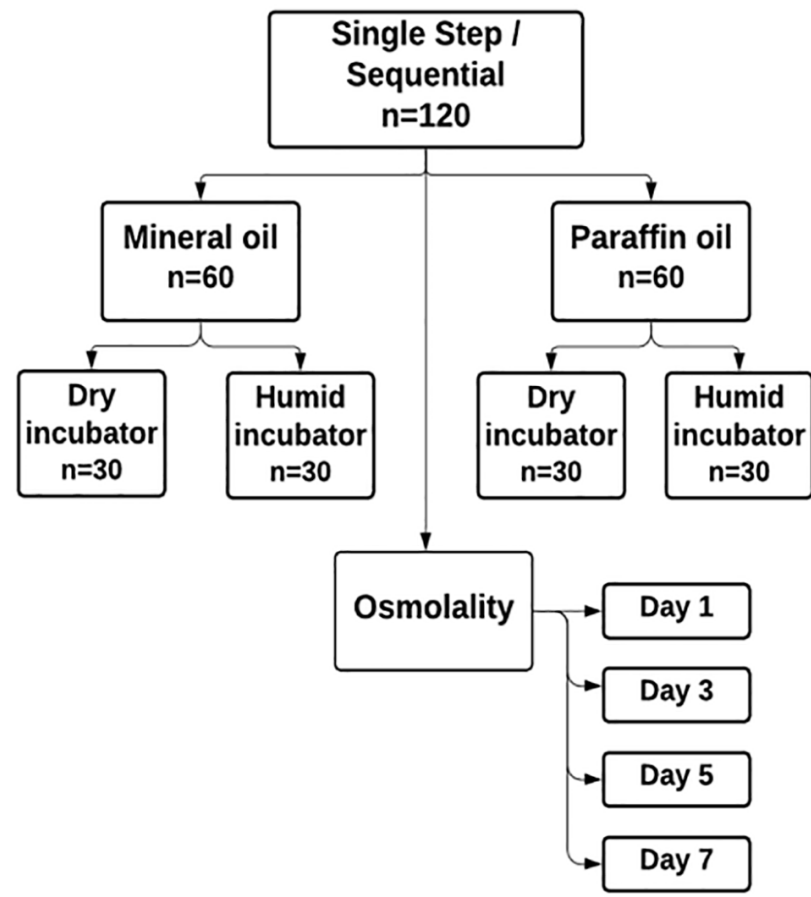

Figure 1. Diagram illustrating the experimental design: 120 dishes each one with 4 droplets of single step medium +4 droplets of sequential medium (total of 960 droplets).
The sample size was calculated considering a $10 \%$ increase in osmolality result as the main variable. Thus, it was calculated that the analysis of 30 samples would provide a power test of 0.80 and alpha power of 0.05 . So, for each day we measured 120 droplets of each culture media and used the mean \pm SD for analysis. Statistical analysis was performed using ANOVA test (SPSS 23.0, IBM) and post hoc Dunn-Sidák. Difference were considered significant at $p<0.05$.

\section{RESULTS}

A total of 960 droplets of culture media from 120 dishes, each one prepared with 4 droplets of CSCM-C and 4 droplets of Cleavage Medium, were evaluated for osmolality measurement, in four different moments. When we compared the osmolality of both culture media, we observed that single step medium (CSCM) had lower osmolality than sequential medium (Cleavage) in all periods, regardless of the type of oil or incubator (Figure 2 and Figure 3). Therefore, we performed a comparison on osmolality rate of change (slope) between different media.

When we compared the osmolality results on day 1 and day 3 , in both culture media, covered with both type oils, we observed a significantly increase in osmolality regardless the type of incubator used, humid or dry.

When we analyzed the osmolality results of the single step culture medium, that were covered with both mineral and paraffin oil and placed in the dry incubator, we observed a significant increase in the results throughout the study time, i.e., D7 $>$ D5 $>$ D3 $(p<0.001$ for both oils) (Table 1$)$. On the other hand, when we made de same comparison with the single step culture medium covered with both mineral and paraffin oil, placed in the humid incubator, the results were similar for all periods, i.e., D3=D5=D7 (Table 1).

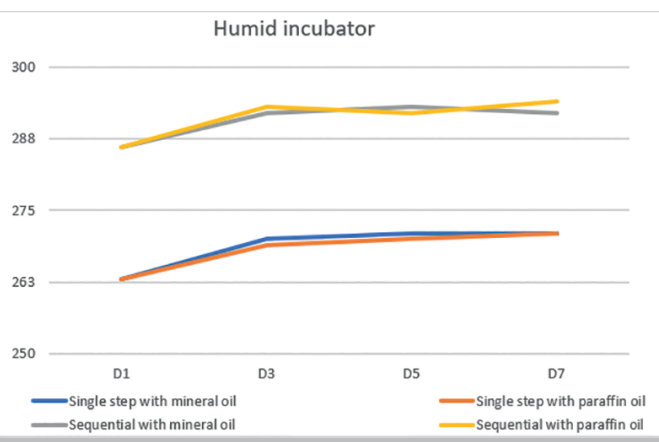

Figure 2. Mean osmolality of Single Step and Sequential media, covered with paraffin and mineral oil, in humid incubator on days $1,3,5$ and 7 .

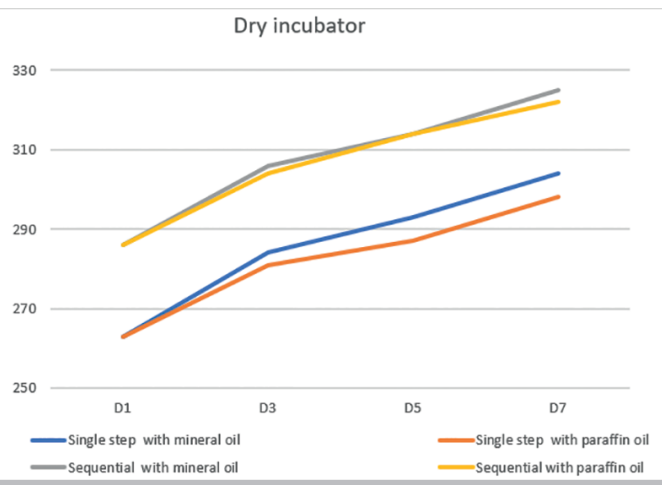

Figure 3. Mean osmolality of Single Step and Sequential media, covered with paraffin and mineral oil, in dry incubator on days $1,3,5$ and 7 . 


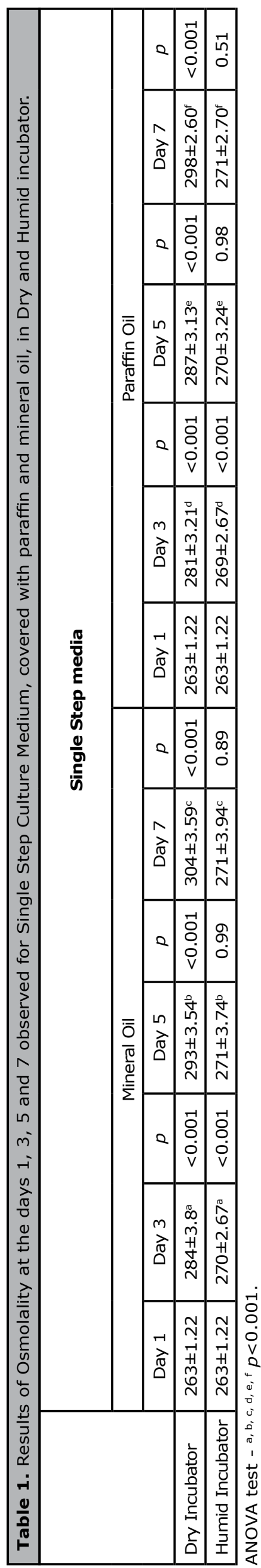

When we analyzed the osmolality results of the sequential medium, that were covered with both mineral and paraffin oil and placed in the dry incubator, we observed a significant increase in the results throughout the study time, i.e., D7 >D5 >D3 ( $p<0.001$ for both oils) (Table 2$)$. On the other hand, when we made de same comparison with sequential medium covered with both mineral and paraffin oil, placed in the humid incubator, the results were similar for all periods, i.e., D3=D5=D7 (Table 2).

When we compared the impact of the different incubators on the culture media osmolality results, we observed that for both culture media, osmolality results were significantly lower in the humid incubators, in all periods, when droplets were covered with both oils (Table 1 and Table 2).

The impact of the different types of oil on the osmolality results was also evaluated and we observed that when the culture media was placed in the humid incubator, minimal variation was detected, in both culture media and in both oils. However, when single step culture medium was placed in the dry incubator, covered with mineral oil, we observed a higher osmolality than the covered with paraffin oil (Table 3 ). For sequential media, a significantly higher osmolality was observed only at day 7 in dry incubator, when covered with mineral oil (Table 4).

\section{DISCUSSION}

Our study demonstrated that humid incubator is better than dry incubator for maintaining the osmolality of culture media, regardless of the type of culture media, as in the humid incubator, the osmolality remained almost unaltered after 7 days. Swain et al. (2016) and Yumoto et al. (2019) also observed an increase of the osmolality in culture media in dry incubator. This fact can be explained as the absence of humidity causes a higher evaporation rate and is independent of the type of oil used.

The mean osmolality, observed on day 1 , of single step medium was $263 \mathrm{mOsm}$ and the mean initial osmolality of sequential medium was $286 \mathrm{mOsm}$. According to Irvine Scientific osmolality ranges to $260-270 \mathrm{mOsm} /$ $\mathrm{kg}$ for CSCM-C and ranges to 285-295 mOsm $/ \mathrm{kg}$ for Cook Cleavage Medium. Swain (2019) observed that an osmolality range of $255-265 \mathrm{mOsm} / \mathrm{kg}$ was ideal for single step media, used in an uninterrupted culture system, considering evaporation that occur in embryo culture. Also, osmolality values higher than $300 \mathrm{mOsm}$, are deleterious for embryo development. Thus, the osmolality obtained initially was in accordance with what was previously described as being optimal.

Osmolality changes may negatively affect embryos mitosis rates over the days in culture, as well as aneuploidies rates (Swain, 2019). Previous studies showed that the osmotic stress could disturbs the meiotic spindle in oocytes. Similar effects may occur in the embryo meiotic spindle, causing aneuploidies or mosaicism (Mullen et al., 2004). Lack of humidity causes a higher evaporation rate of the culture media and could be an additional source of stress to embryos. Changes in $\mathrm{pH}$ were also related to osmolality changes as $\mathrm{pH}$ depends on amount of water, salts, aminoacids, proteins, buffers and the incubator $\mathrm{CO} 2$ levels. Since the osmolality is compromised water evaporation, $\mathrm{pH}$ is also compromised (Swain, 2018; Gardner \& Kelley, 2017). Other studies have also demonstrated the clinical relevance of the humidity in human embryo development. Fawzy et al. (2017) described lower clinical pregnancy rate when embryos were cultured in dry incubators when compared to those cultured in humid incubators. Del Gallego et al. (2018) observed a higher blastocyst formation after culture in humid conditions when compared to dry conditions.

In addition, incubator oxygen level is crucial for better results in IVF cycles (Catt \& Henman, 2000). Low oxygen concentration may enhance blastocyst development (Gardner \& Lane, 1997; Kovačič, 2012; Bontekoe et al., 


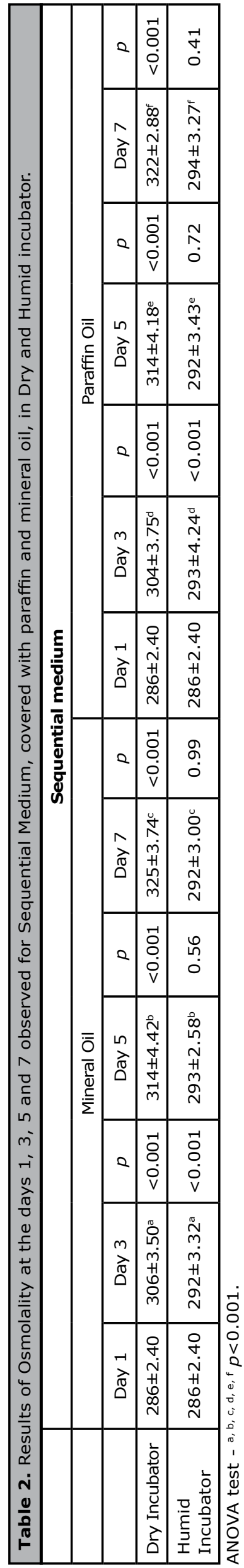

2012). Guo et al. (2014) had significantly higher blastocyst rates when embryos were cultivated in incubators with $5 \%$ oxygen compared to the group with $20 \%$ of oxygen. Furthermore, incubators with $5 \%$ of oxygen obtained better results by reducing oxidation levels and maintain the integrity of the amino acids in culture media (Biggers et al., 2004; Tarahomi et al., 2019). Therefore, in our study we used only $5 \%$ oxygen incubators, dry or humid.

Oil is commonly used in embryo culture to minimize evaporation, fluctuations on $\mathrm{pH}$ and temperature of media (Otsuki et al., 2009; Labied et al., 2019). The type of oil used, directly affects the speed of evaporation: heavy oils provide less evaporation compared to a lighter oil (Swain, 2018). Our study showed that in dry incubator paraffin oil provided a greater protection against the evaporation to single step media on day 3, 5 and 7, which is essential for a successfully uninterrupted culture system. No significant difference was observed in sequential media on days 3 and 5 . On day 7 , the paraffin oil was better to maintain osmolality level. However, these media must be replaced by a proper media on day 3 of development. We measured osmolality of sequential media until day 7 as control to single step media. Swain (2018) also showed that heavier oil, provided a greater protection against the evaporation of media, if compared to the lighter oil in dry incubator. Moreover, it is important to note that osmolality had a significantly increased from day 1 until day 3 in both media, covered with both types of oils regardless the type if incubator used, probably due to media equilibration that occurs inside the incubators.

Oil composition is also an important factor that can affect IVF outcomes. Mineral oil is more likely to suffer photooxidation and peroxidation due unsatureted bonds which can lead to worse fertilization and embryo development (Otsuki et al., 2007). Sifer et al. (2009) compared commercial oils used in human embryo culture and showed that Ovoil promoted more top-quality embryos on day 3 than other mineral oil in humid conditions. Martinez et al. (2017) demonstrated that mineral oil affected negatively the cleavage rate of the embryo and the speed of the blastocyst formation, if compared with paraffin oil, probably due osmolality and $\mathrm{pH}$ changes. Nevertheless, Labied et al. (2019) analyzed embryos from dishes covered with paraffin and mineral oil and no differences were observed in top quality embryos $(41.7 \% \times 41.2 \%)$, utilization rates $(92.2 \%$ $\times 92.0 \%)$ and livebirth rates $(26.9 \% \times 26.2 \%)$. However, in this study they do not describe the type of incubator, humid or dry, used for embryo culture.

The type of culture medium might also have an impact on quality of the embryos generated from assisted reproduction techniques (Mantikou et al., 2013; Sepúlveda et al., 2009). Moreover, optimal performance of culture media depends on oil and incubator type used (Sifer et al., 2009). Nonetheless there is no consensus regarding of which culture media provides a higher livebirth rate (Sfontouris et al., 2016; Dieamant et al., 2017). Therefore, we performed all analysis using two different culture media, single step and sequential, in order to evaluate the possible effect of the different incubators and oils for both types. Our results demonstrated that, although single step media have lower osmolality, the variation observed through the study time was similar to the observed with the sequential medium

It is of fundamental importance how the dishes were prepared as Swain et al. (2012) observed an increase in osmolality when the temperature was $37^{\circ} \mathrm{C}$. They also reported that $10 \mu \mathrm{l}$ droplets of culture medium have higher osmolality when compared to 20 or $40 \mu \mathrm{l}$ droplets. As all dishes were prepared by the same person, using the same material, equipment, calibration and temperature, at the same time of the day and the same volume of culture medium and oil, we did avoid any preparation bias that could interfere with the results. 


\begin{tabular}{|c|c|c|c|c|c|c|}
\hline \multirow{2}{*}{ Single Step Medium } & \multicolumn{3}{|c|}{ Dry incubator } & \multicolumn{3}{|c|}{ Humid incubator } \\
\hline & Mineral Oil & Paraffin Oil & $p$ & Mineral Oil & Paraffin Oil & $p$ \\
\hline Day 1 & $263 \pm 1.22$ & $263 \pm 1.22$ & 1 & $263 \pm 1.22$ & $263 \pm 1.22$ & 1 \\
\hline Day 3 & $284 \pm 3.84$ & $281 \pm 3.21$ & $<0.001$ & $270 \pm 2.67$ & $269 \pm 2.67$ & 0.39 \\
\hline Day 5 & $293 \pm 3.54$ & $287 \pm 3.13$ & $<0.001$ & $271 \pm 3.74$ & $270 \pm 3.24$ & 0.98 \\
\hline Day 7 & $304 \pm 3.59$ & $298 \pm 2.60$ & $<0.001$ & $271 \pm 3.94$ & $271 \pm 2.70$ & 0.99 \\
\hline
\end{tabular}

ANOVA Test

Table 4. Results of Osmolality at the days 1, 3,5 and 7 observed for Sequential Medium, covered with mineral and paraffin oil, in Dry and Humid incubator.

\begin{tabular}{|l|c|c|c|c|c|c|}
\hline Sequential Medium & \multicolumn{3}{|c|}{ Dry incubator } & \multicolumn{3}{c|}{ Humid incubator } \\
\hline & Mineral Oil & Paraffin Oil & $p$ & Mineral Oil & Paraffin Oil & $p$ \\
\hline Day & $286 \pm 2.4$ & $286 \pm 2.4$ & 1 & $286 \pm 2.4$ & $286 \pm 2.4$ & 1 \\
\hline Day 3 & $306 \pm 3.5$ & $304 \pm 3.75$ & 0.21 & $292 \pm 3.32$ & $293 \pm 4.24$ & 0.76 \\
\hline Day 5 & $314 \pm 4.42$ & $314 \pm 4.18$ & 1 & $293 \pm 2.58$ & $292 \pm 3.43$ & 0.91 \\
\hline Day 7 & $325 \pm 3.74$ & $322 \pm 2.88$ & 0.003 & $292 \pm 3.00$ & $294 \pm 3.27$ & 0.62 \\
\hline
\end{tabular}

ANOVA Test

In conclusion, our study demonstrated that humid incubator is better for maintaining osmolality and paraffin oil protect single step media from evaporation in dry incubator, which can improve continuous and undisturbed embryonic culture.

\section{Declarations of interest: none}

\section{CONFLICT OF INTEREST}

The authors declare no conflict of interest.

\section{Corresponding author:}

Renata de Lima Bossi

Centro de Medicina Reprodutiva - ORIGEN

Horizonte/MG- Brazil

E-mail: renatabossi@gmail.com

\section{REFERENCES}

Biggers JD, Racowsky C. The development of fertilized human ova to the blastocyst stage in $\mathrm{KSOM}(\mathrm{AA})$ medium: is a two-step protocol necessary? Reprod Biomed Online. 2002;5:133-40. PMID: 12419037 DOI: 10.1016/S14726483(10)61615-X

Biggers JD, McGinnis LK, Lawitts JA. Enhanced effect of glycyl-L-glutamine on mouse preimplantation embryos in vitro. Reprod Biomed Online. 2004;9:59-69. PMID: 15257821 DOI: $10.1016 / S 1472-6483(10) 62111-6$

Bolton VN, Cutting $\mathrm{R}$, Clarke $\mathrm{H}$, Brison DR. ACE consensus meeting report: culture systems. Hum Fertil (Camb). 2014;17:239-51. PMID: 25116334 DOI: $10.3109 / 14647273.2014 .944417$

Bontekoe S, Mantikou E, van Wely M, Seshadri S, Repping $\mathrm{S}$, Mastenbroek S. Low oxygen concentrations for embryo culture in assisted reproductive technologies. Cochrane Database Syst Rev. 2012;CD008950. PMID: 22786519 DOI: $10.1002 / 14651858 . C D 008950 . p u b 2$
Catt JW, Henman M. Toxic effects of oxygen on human embryo development. Hum Reprod. 2000;15:199-206. PMID: 11041525 DOI: 10.1093/humrep/15.suppl_2.199

Del Gallego R, Albert C, Marcos J, Larreategui Z, Alegre L, Meseguer $M$. Humid vs. dry embryo culture conditions on embryo development: a continuous embryo monitoring assessment. Fertil Steril. 2018;110:e362-3. DOI: 10.1016/j. fertnstert.2018.07.1012

Dieamant F, Petersen CG, Mauri AL, Comar V, Mattila M, Vagnini LD, Renzi A, Petersen B, Ricci J, Oliveira JBA, Baruffi RLR, Franco JG Jr. Single versus sequential culture medium: which is better at improving ongoing pregnancy rates? A systematic review and meta-analysis. JBRA Assist Reprod. 2017;21:2406. PMID: 28837034 DOI: 10.5935/1518-0557.20170045

Eaton JL, Lieberman ES, Stearns C, Chinchilla M, Racowsky C. Embryo culture media and neonatal birthweight following IVF. Hum Reprod. 2012;27:375-9. PMID: 22128298 DOI: $10.1093 /$ humrep/der381

Elder K, van den Bergh M, Woodward B, eds. Troubleshooting and problem-solving in the IVF laboratory. Cambridge: Cambridge University Press; 2015.

Erstad BL. Osmolality and osmolarity: narrowing the terminology gap. Pharmacotherapy. 2003;23:1085-6. PMID: 14524639 DOI: $10.1592 /$ phco.23.10.1085.32751

Fawzy M, AbdelRahman MY, Zidan MH, Abdel Hafez FF, Abdelghafar $\mathrm{H}$, Al-Inany $\mathrm{H}$, Bedaiwy MA. Humid versus dry incubator: a prospective, randomized, controlled trial. Fertil Steril. 2017;108:277-83. PMID: 28651960 DOI: 10.1016/j.fertnstert.2017.05.036

Gardner DK, Lane M. Culture and selection of viable blastocysts: a feasible proposition for human IVF? Hum Reprod Update. 1997;3:367-82. PMID: 9459282 DOI: 10.1093/ humupd/3.4.367 
Gardner DK, Kelley RL. Impact of the IVF laboratory environment on human preimplantation embryo phenotype. J Dev Orig Health Dis. 2017;8:418-35. PMID: 28610639 DOI: $10.1017 / S 2040174417000368$

Guo N, Li Y, Ai J, Gu L, Chen W, Liu Q. Two different concentrations of oxygen for culturing precompaction stage embryos on human embryo development competence: a prospective randomized sibling-oocyte study. Int J Clin Exp Pathol. 2014;7:6191-8. PMID: 25337269

Heitmann RJ, Hill MJ, James AN, Schimmel T, Segars JH, Csokmay JM, Cohen J, Payson MD. Live births achieved via IVF are increased by improvements in air quality and laboratory environment. Reprod Biomed Online. 2015;31:36471. PMID: 26194882 DOI: 10.1016/j.rbmo.2015.04.011

Labied S, Jouan C, Wenders F, Ravet S, Gaspard O, Thonon F, Gridelet V, Henry L, Perrier d'Hauterive S, Nisolle $M$. Comparison between paraffin and mineral oil covering on early human embryo culture: a prospective randomized study. Syst Biol Reprod Med. 2019;65:81-6. PMID: 29985652 DOI: 10.1080/19396368.2018.1492645

Mantikou E, Youssef MA, van Wely M, van der Veen F, Al-Inany HG, Repping S, Mastenbroek S. Embryo culture media and IVF/ICSI success rates: a systematic review. Hum Reprod Update. 2013;19:210-20. PMID: 23385469 DOI: 10.1093/humupd/dms061

Martinez CA, Nohalez A, Parrilla I, Motas M, Roca J, Romero I, García-González DL, Cuello C, Rodriguez-Martinez H, Martinez EA, Gil MA. The overlaying oil type influences in vitro embryo production: differences in composition and compound transfer into incubation medium between oils. Sci Rep. 2017;7:10505. PMID: 28874873 DOI: 10.1038/s41598-017-10989-5

Morbeck DE, Krisher RL, Herrick JR, Baumann NA, Matern $D$, Moyer T. Composition of commercial media used for human embryo culture. Fertil Steril. 2014;102:759-66.e9. PMID: 24998366 DOI: 10.1016/j.fertnstert.2014.05.043

Mori C, Kuwayama M, Silber SJ, Kagawa N, Takehara Y, Kato $O$. Water evaporation and osmolarity change of human embryo culture media in humid or in dry culture systems. Fertil Steril. 2010;94:s151. DOI: 10.1016/j.fertnstert.2010.07.607

Mullen SF, Agca $Y$, Broermann DC, Jenkins $C L$, Johnson CA, Critser JK. The effect of osmotic stress on the metaphase II spindle of human oocytes, and the relevance to cryopreservation. Hum Reprod. 2004;19:1148-54. PMID: 15070883 DOI: $10.1093 /$ humrep/deh201

Otsuki J, Nagai Y, Chiba K. Peroxidation of mineral oil used in droplet culture is detrimental to fertilization and embryo development. Fertil Steril. 2007;88:741-3. PMID: 17292894 DOI: $10.1016 /$ j.fertnstert.2006.11.144

Otsuki J, Nagai Y, Chiba K. Damage of embryo development caused by peroxidized mineral oil and its association with albumin in culture. Fertil Steril. $2009 ; 91: 1745-9$. PMID: 18440508 DOI: $10.1016 /$ j.fertnstert.2008.03.001

Richards T, Wang F, Liu L, Baltz JM. Rescue of postcompaction-stage mouse embryo development from hypertonicity by amino acid transporter substrates that may function as organic osmolytes. Biol Reprod. 2010;82:769-77. PMID: 20018907 DOI: 10.1095/biolreprod.109.081646
Sepúlveda S, Garcia J, Arriaga E, Diaz J, Noriega-Portella $L$, Noriega-Hoces L. In vitro development and pregnancy outcomes for human embryos cultured in either a single medium or in a sequential media system. Fertil Steril. 2009;91:1765-70. PMID: 18439587 DOI: $10.1016 / \mathrm{j}$. fertnstert.2008.02.169

Sfontouris IA, Martins WP, Nastri CO, Viana IG, Navarro $P A$, Raine-Fenning N, van der Poel S, Rienzi L, Racowsky C. Blastocyst culture using single versus sequential media in clinical IVF: a systematic review and meta-analysis of randomized controlled trials. J Assist Reprod Genet. 2016;33:1261-72. PMID: 27491772 DOI: 10.1007/ s10815-016-0774-5

Sifer C, Pont JC, Porcher R, Martin-Pont B, Benzacken $B$, Wolf JP. A prospective randomized study to compare four different mineral oils used to culture human embryos in IVF/ICSI treatments. Eur J Obstet Gynecol Reprod Biol. 2009;147:52-6. PMID: 19615809 DOI: 10.1016/j. ejogrb.2009.06.023

Swain JE, Cabrera L, Xu X, Smith GD. Microdrop preparation factors influence culture-media osmolality, which can impair mouse embryo preimplantation development. Reprod Biomed Online. 2012;24:142-7. PMID: 22197605 DOI: $10.1016 /$ j.rbmo.2011.10.008

Swain JE, Schoolcraft WB, Bossert N, Batcheller AE. Media osmolality changes over 7 days following culture in a non-humidified benchtop incubator. Fertil Steril. 2016;106:e362. DOI: 10.1016/j.fertnstert.2016.07.1028

Swain JE. Decisions for the IVF laboratory: comparative analysis of embryo culture incubators. Reprod Biomed Online. 2014;28:535-47. PMID: 24656561 DOI: 10.1016/j. rbmo.2014.01.004

Swain JE. Different mineral oils used for embryo culture microdrop overlay differentially impact media evaporation. Fertil Steril. 2018;109:e53. DOI: 10.1016/j.fertnstert.2018.02.101

Swain JE. Controversies in ART: can the IVF laboratory influence preimplantation embryo aneuploidy? Reprod Biomed Online. 2019;39:599-607. PMID: 31492521 DOI: 10.1016/j.rbmo.2019.06.009

Tarahomi M, Vaz FM, van Straalen JP, Schrauwen FAP, van Wely M, Hamer G, Repping S, Mastenbroek S. The composition of human preimplantation embryo culture media and their stability during storage and culture. Hum Reprod. 2019;34:1450-61. PMID: 31348827 DOI: 10.1093/humrep/dez102

Yumoto K, Iwata K, Sugishima M, Yamauchi J, Nakaoka M, Matsumoto I, Moriwaki H, Shimura T, Mio Y. Mineral oil viscosity affects the osmotic pressure of human embryonic culture medium microdrops in non-humidified incubators. Fertil Steril. 2018;110:e52. DOI: 10.1016/j. fertnstert.2018.07.161

Yumoto K, Iwata K, Sugishima M, Yamauchi J, Nakaoka M, Tsuneto M, Shimura T, Flaherty S, Mio Y. Unstable osmolality of microdrops cultured in non-humidified incubators. J Assist Reprod Genet. 2019;36:1571-7. PMID: 31267335 DOI: $10.1007 / \mathrm{s} 10815-019-01515-9$ 